\title{
Effect of Magnification on the Precision of Indoor Telescope Pointing
}

\author{
By Francis E. Washer
}

\begin{abstract}
The principle variables affecting the precision of telescope pointing at an indoor target are magnification, brightness, and vernier acuity of the observer's eye. A series of experiments were performed in which magnification was the principle variable. All observations were made by a single observer, thus reducing variation in the vernier acuity. The brightness of the background against which the target was viewed was maintained at approximately the same level throughout the experiments to minimize effects arising from variation of brightness. Under these conditions, the relation between probable error of a single pointing $\left(P E_{\mathrm{s}}\right)$ and magnification $(M)$ is approximately $P E_{\mathrm{s}}$ (in seconds) $=\frac{4.962}{M}+0.068$.
\end{abstract}

\section{Introduction}

This study of the factors affecting the precision of telescope pointing was initiated in connection with the Range and Height Finder Development Project sponsored at the Bureau by the United States Army Ordnance. As a range finder is essentially a double-pointing instrument, it is possible to deduce the error that may be ascribed to the purely optical phase of the range finder system from the results obtained with a single telescope. The part of this investigation that deals with the precision of pointing for outdoor targets has already been reported $[1,2]^{1}$

In this earlier investigation, no consideration was given to factors that may affect the probable error of a single pointing $\left(P E_{s}\right)$ other than the distance separating target and observer. Other workers in this field have, however, devoted a great deal of attention to the correlation of $P E_{s}$ with magnification of the observing telescope. The results of these attempts showed such wide discrepancies from one observer to another that it seemed worthwhile to investigate this matter further under carefully controlled conditions.

As control of conditions for outdoor pointing is not practicable, and as experience has shown that low and consistent values of $P E_{s}$ are obtained indoors, the present study is made for indoor

\footnotetext{
${ }^{t}$ Figures in brackets indicate the literature references at the end of this paper.
}

targets only. It must be emphasized that the term "pointing," as used in this paper, refers to a type of pointing where all mechanical errors have been eliminated and the error of pointing found is an attribute of the combination of the optical system and observer only, and is wholly devoid of such errors that may result from incorrect reading of verniers and scales such as exist on transit circles and similar mechanical devices. In addition, the term $P E_{s}$ refers to the probable error of a single pointing about the instantaneous "true" pointing at the time at which it is made, and is a measure of the error of a single pointing determined from a number of pointings taken rapidly, and does not contain any appreciable effect of drift.

In planning an investigation into the effect of magnification on pointing accuracy, it is clear that there are many variables that must either be controlled or allowed to affect the results in whatever manner they may. For example, if the target and reticle size are kept unchanged and the magnification varied then the apparent width of target and reticle increases directly as the magnification. That this change in apparent width may be expected to have some effect is evidenced by some work done by Guild dealing with errors of optical settings [3]. His work was done with the unaided eye and showed appreciable variation for crossline widths varying from 10 to 180 seconds with the greatest change taking place between 10 and 


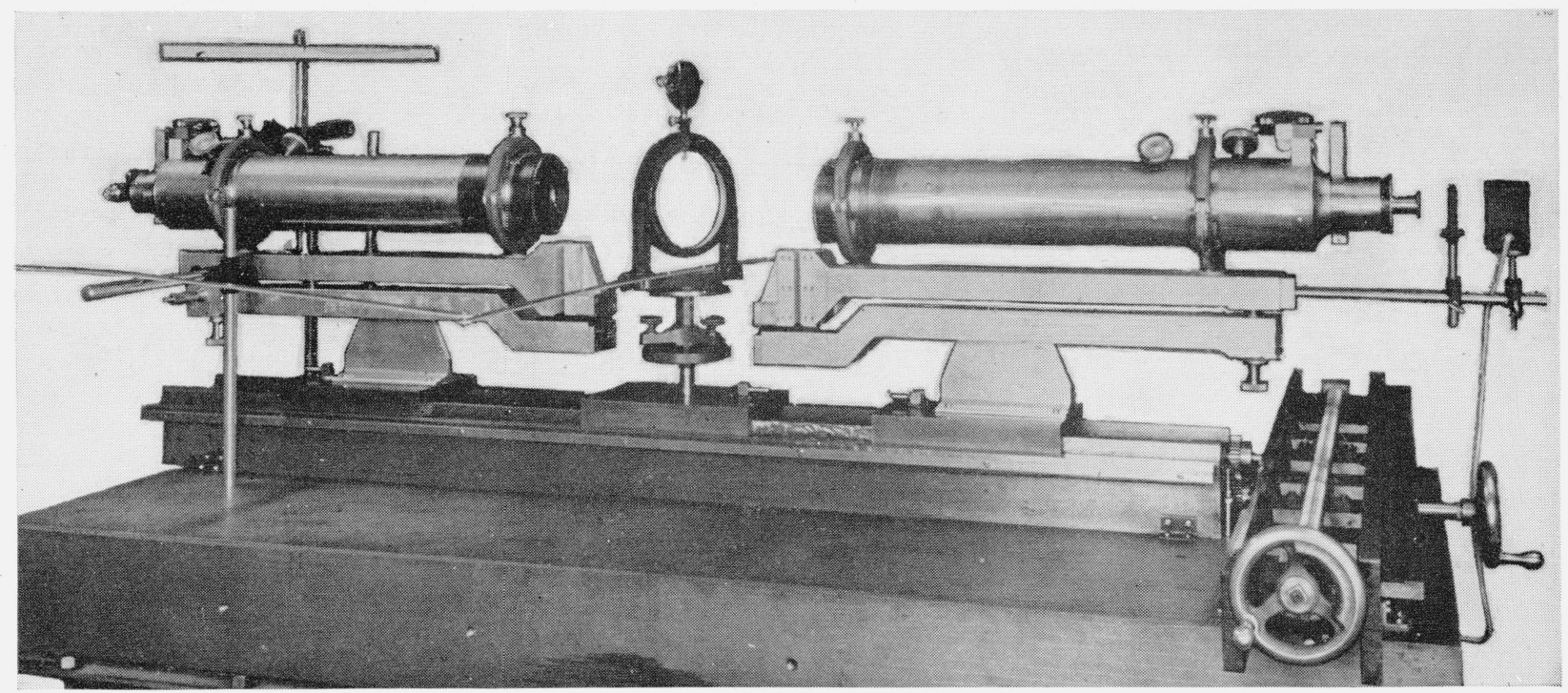

Figure 1.-Arrangement of telescope, collimator, and rotating prism as used in the experiment.

40 seconds. It is therefore possible that the value of $P E_{s}$ for low magnifications may be influenced somewhat by the variation of apparent line widths. However, it was decided to omit control of this factor because the major portion of a given series of observations is taken under conditions where the apparent line width is over 40 seconds and hence in the region of very slow change.

A second factor that may affect the probable error of pointing is the apparent brightness of the background against which the target is viewed. This effect is discussed by Pelzer [4], who ascribes to it an importance out of proportion to its effect, in the opinion of the present author. This opinion is based on unpublished work in which it is found that while there is a slow increase in $P E_{s}$ as one proceeds from a brightness so high as to be barely tolerable to a brightness lower by a factor of 1,000 , the magnitude of this change can be neglected as long as the brightness is maintained at a level compatible with easy seeing. At very low brightnesses, there is a rapid increase in $P E_{s}$ with diminishing brightness. However, this second factor was controlled during the present experiments so that the apparent brightness of the background against which the target is viewed was kept approximately constant at a level of easy seeing throughout this part of the work.

\section{Apparatus and Method of Measurement}

The apparatus and method of measurement are essentially the same as those described in the study of outdoor pointing accuracy [1]. The differences are primarily those concerned with size of telescope and auxiliary equipment. Two telescopes are used, one having an objective of 711-mm focal length and $100-\mathrm{mm}$ aperture, and the second having an objective of 1,143-mm focal length and $50-\mathrm{mm}$ aperture. The arrangement for the telescope with objective of 711-mm focal length is shown in figure 1. The collimator has an objective of 711-mm focal length and 100-mm aperture. The weak prism has $150-\mathrm{mm}$ clear aperture, and the angle between its surfaces is 4 minutes.

To vary the pointing without disturbing the viewing telescope, the weak prism is placed in front of the telescope objective and so mounted that it may be rotated about a vertical axis, which is also parallel to the prism axis. As the deviation of a parallel beam of light caused by a weak prism is a function of the angle of incidence on the prism surface, the image of the target can be caused to move from side-to-side of the telescope cross-hair intersection by oscillating the prism. During the course of an observation the prism is rotated until 
the image of the target appears to coincide with the cross-hair in the telescope, and the angular position of the prism is noted with the aid of an auxiliary telescope and scale in conjunction with a mirror attached to the rotating prism. This reading is converted into seconds in the object space. A series of ten such observations is taken and the probable errors, $P E_{s}$, determined from these data. Five such 10-groups are usually taken in a single run with a short intermission between each 10-group, and the average $P E_{s}$ is accepted as the value of $P E_{s}$ for the run.

All observations and the recording of data are performed by a single observer, the author, which, together with the fact that the rotation of the prism is controlled by a smooth rod that must be released between observations, acts to reduce any effect of memory in making successive settings, and so tends to ensure the independence of successive observations.

The probable error of a single pointing, $P E_{s}$, is computed from the approximate formula

$$
\left.P E_{s} \text { (in seconds }\right)=\frac{0.8453 k \sum_{1}^{n}\left|x_{i}-\bar{x}\right|}{\sqrt{n(n-1)}},
$$

where $x_{1}, x_{2}, \ldots x_{i} \ldots x_{n}$ denote the $n$ observations of a group and $\bar{x}=\frac{\sum x_{i}}{n}$ is the arithmetic mean of the $n$ readings, and $k$ is the calibration constant of the prism for conversion of millimeters observed on the scale to seconds deviation of the light beam caused by the prism. The observations are considered 10 at a time, and for $n=10$, eq 1 can be written

$$
P E_{s}=0.0891 k \sum_{1}^{10}\left|x_{i}-\bar{x}\right| .
$$

The magnifications, $6,12,25.5$, and 48 are obtained by four ocular lenses specially designed for use with the 711-mm telescope objective. Other magnifications, for the most part greater, are obtained by using a compound microscope as an ocular. By suitable choice of microscope objectives and oculars a wide range of magnifications is provided. The magnifications of the telescope with the four oculars are determined by two methods, first by the ratio of the focal length of the telescope objective to that of the ocular, and second by the ratio of entrance pupil to exit pupil with the telescope focused for parallel light, a condition that prevails throughout the experiment. For the four oculars the agreement by these two methods is good.

When microscopes are used as oculars, the total telescope magnification, $M$, is computed from the relation $M=m_{1} \times m_{2}$, where $m_{1}$ is the objective magnification and $m_{2}$ the magnifying power of the microscope. The value of $m_{1}$ is given by the ratio of the focal length of the telescope objective to the distance of distinct vision, $250 \mathrm{~mm}$. To insure against the errors that may result from accepting the nominal values of magnifying power of a microscope, each of the values of $m_{2}$ is carefully determined by experiment.

\section{Results of Measurements}

\section{Spider Fiber as Target and 711-mm Objective in Viewing Telescope}

The target is a spider fiber mounted in a vertical position in the focal plane of the collimator. The diameter of the spider fiber is approximately $4 \mu$; accordingly its diameter subtends an angle of approximately 1.3 seconds at the objective of the collimating telescope. This value of 1.3 seconds is nearly equal to the resolving power (1.2 seconds) of the observing telescope. The bright background, against which the spider fiber is viewed, is provided by a ground glass screen illuminated by a $6-v$ projection lamp. The brightness of the screen is varied when necessary by interposing neutral filters between the lamp and screen. The reticle in the observing telescope consists of two platinum wires $0.0001 \mathrm{in}$. in diameter intersecting at 90 degrees, this reticle being oriented to present the appearance of an $X$. For the magnifications used, the apparent angular width of the spider hair ranges from approximately 8 to 329 seconds, while the apparent angular width of the reticle wires ranges from 5 to 227 seconds.

The results of measurement are listed in table 1 and shown graphically in figure 2. The form of the curve of $P E_{s}$ versus magnification is an hyperbola, as is shown in figure 3 , by plotting $P E_{s}$ against the reciprocal of magnification. The straight line curve so obtained has a definite intercept on the $P E_{s}$ axis at $\frac{1}{M}=0$ instead of passing through zero as might be expected. 


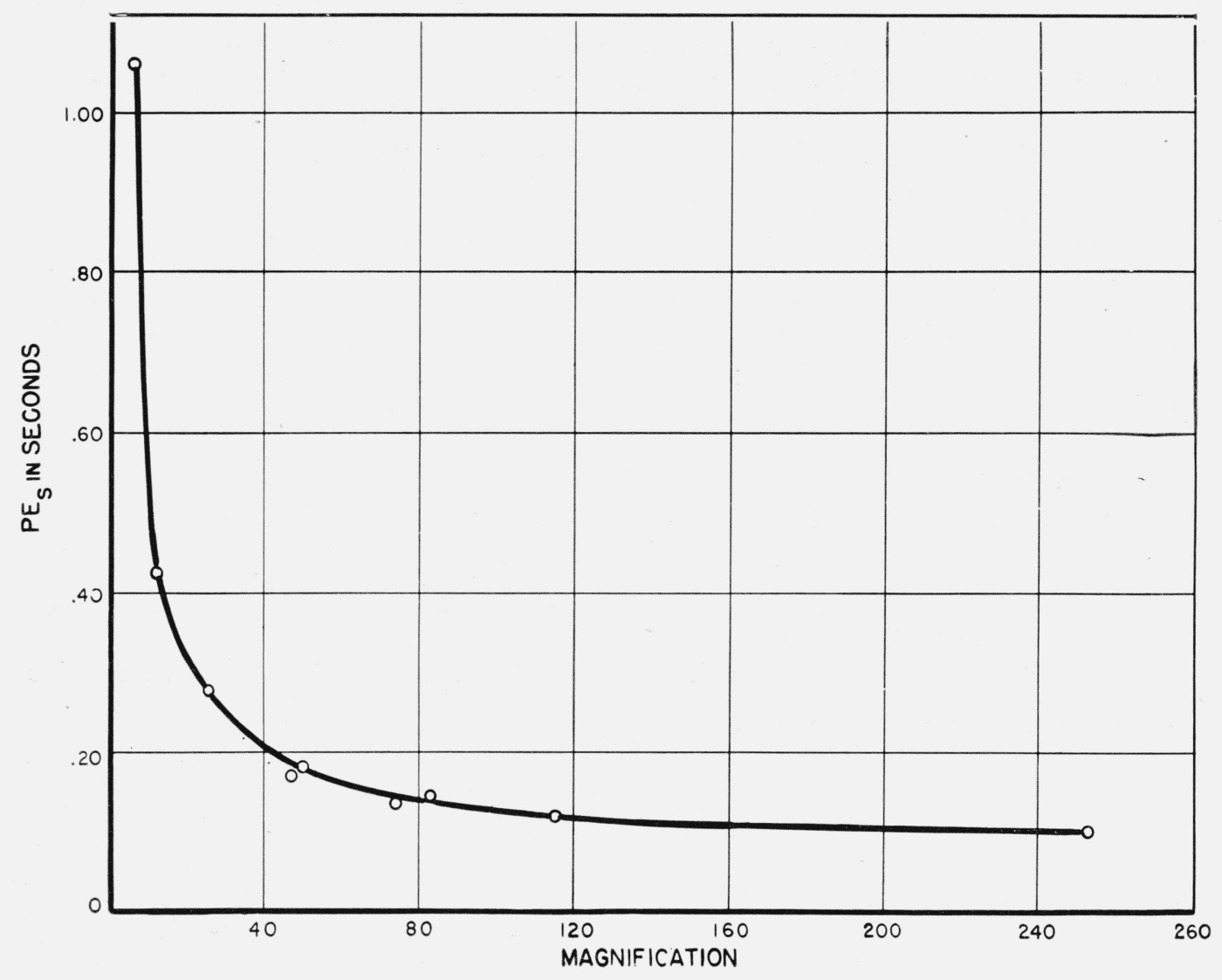

Figure 2.-$P E_{s}$ versus magnification.

The results are for a viewing telescope with an objective of $711-\mathrm{mm}$ focal length, 100-mm aperture. A spider fiber of approximately $4 \mu$ diameter mounted in the focal plane of the collimator is the target.

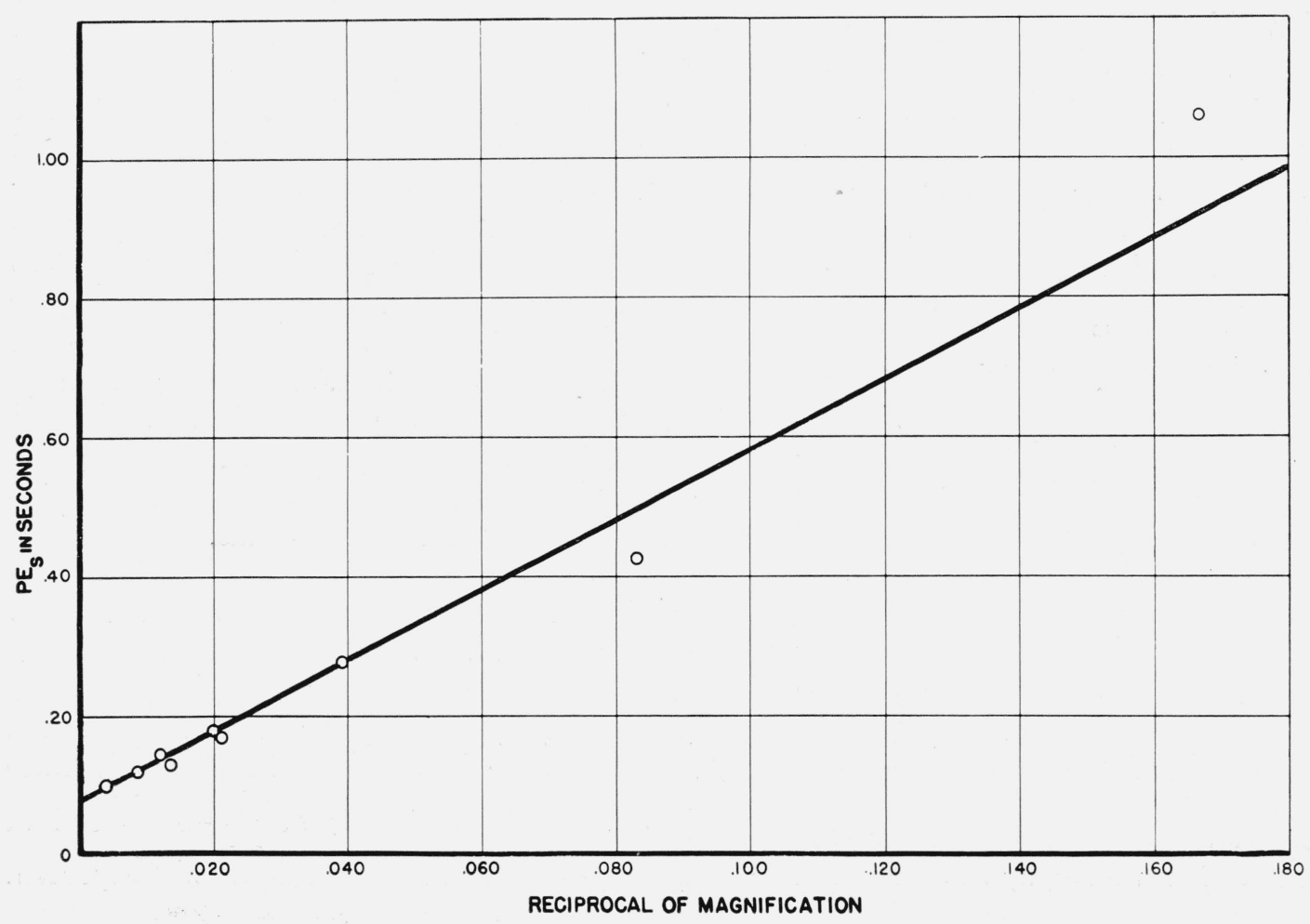

Figure $3 .-P E_{s}$ versus reciprocal of magnification.

The results are for a viewing telescope with an objective of 711-mm focal length, $100-\mathrm{mm}$ aperture. A spider fiber of approximately $4 \mu$ diameter mounted in the focal plane of the collimator is the target. 
Thus the relation connecting $P E_{s}$ and magnification is of the form

$$
P E_{s}=\frac{A}{M}+B,
$$

where $A$ and $B$ are constants, and $M$ is the magnification. For the curve in figure $3, A$ is the slope of the curve, and $B$, the intercept on the $P E_{s}$ axis. Although the values of $A$ and $B$ can be obtained directly from the curve, a more accurate evaluation is made by least-squares considering only those values of $P E_{s}$ for $M=25.5$. The final equation is found to be

$$
P E_{s}=\frac{5.033}{M}+0.076 \text {. }
$$

The values of $P E_{s}$ for magnifications 6 and 12 are omitted from the solution because they do not lie as near to the straight line curve as do the others. Moreover a least-squares solution, based on all observed values of $P E_{s}$, yields computed values of $P E_{s}$ that in two instances differ from the observed values by four or more probable errors. The present solution yields computed values of $P E_{s}$ that in only one instance differ from the observed values of $P E_{s}$ by as much as three probable errors, and that instance is for magnification 12, which was not included in the solution.

These differences between observed values and those calculated from eq 4 are given in table 1 under the heading $\triangle P E_{s}$. It is noteworthy that these values of $\triangle P E_{s}$ are approximately the same or less than the values of $P_{p}$, listed under the column headed 1 , for all values of $M \geqslant 25.5$. Even for magnifications 6 and 12 , the values of $\triangle P E_{s}$ are only 2 or 3 times the value of $P_{p}$. It can therefore be stated that the values of $P E_{s}$ calculated from eq 4 , give excellent agreement with the observed values for the range of magnifications used.

Three values of $P_{p}$ are listed in the tables under the headings 1, 2, and 3 . The values listed under 1 are obtained from the formula

$$
P_{p}=\frac{ \pm 2 P E_{s}}{3 \sqrt{2(n-1)}}
$$

where $n$ is the total number of observations. For the present work this expression is sufficiently well approximated by the formula

$$
P_{p}=\frac{ \pm P E_{s}}{2 \sqrt{n}}
$$

TABLE 1.-Probable error of a single pointing $\left(P E_{s}\right)$ as a function of magnification $(M)$

\begin{tabular}{|c|c|c|c|c|c|c|c|}
\hline \multirow{2}{*}{ Magnification } & \multicolumn{2}{|c|}{$P E_{s}$} & \multirow{2}{*}{ 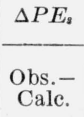 } & \multicolumn{3}{|c|}{$P_{p}$} & \multirow{2}{*}{$\begin{array}{l}\text { Num- } \\
\text { ber } \\
\text { of } 10- \\
\text { groups }\end{array}$} \\
\hline & Obs & Calc & & 1 & 2 & 3 & \\
\hline Diameters & sec & sec & $\sec$ & $\sec$ & sec & $s e c$ & \\
\hline 6 & 1.060 & 0.915 & 0.145 & 0.075 & 0.075 & 0.058 & 5 \\
\hline $12 \ldots$ & 0.425 & .495 & -.070 & .021 & .030 & .043 & 10 \\
\hline $25.5 \ldots \ldots$ & .277 & .273 & .004 & .009 & .020 & .025 & 45 \\
\hline $48 \ldots$ & .170 & .181 & -.011 & .012 & .012 & .004 & 5 \\
\hline $50 \ldots$ & .180 & .177 & .003 & .009 & .013 & .012 & 10 \\
\hline $74 \ldots$ & .135 & .144 & -.009 & .007 & .010 & .017 & 10 \\
\hline $83 \ldots$ & .145 & .137 & .008 & .007 & .010 & .014 & 10 \\
\hline 115 & .120 & .120 & .000 & .008 & .008 & .004 & 5 \\
\hline $253 \ldots \ldots$ & .100 & .096 & .004 & .005 & .007 & .007 & 10 \\
\hline
\end{tabular}

The results are for a viewing telescope with an objective of 711-mm focal length, $100-\mathrm{mm}$ aperture. A spider fiber of approximately $4-\mu$ diameter mounted in the focal plane of the collimator is the target.

The values of $P_{p}$, obtained by this formula, with $n$ taken as the total number of observations, is especially useful for comparison with $\triangle P E_{s}$, the difference between observed and calculated values of $P E_{s}$. The values of $P_{p}$, listed under 2 , are obtained by use of the same formula, with $P E_{s}$ the average value for five 10-groups and for $n=50$. Where there are several 50 -sets involved, the value of $P_{p}$ is the average for the several 50sets, and represents what might be termed the average theoretical probable error of the probable error for five 10 -groups. The value of $P_{p}$, listed under column 3 , is obtained by considering each $P E_{s}$ from a single 10-group as an observation and computing the probable error of the mean for the average value of $P E_{s}$ from five 10 -groups. Where more than one 50 -set is considered, the value of $P_{p}$ is the average for the several 50 -sets. Ideally $P_{p}$, computed in this manner, should equal the value of $P_{p}$ listed under column 2 . It is noteworthy that fairly good agreement prevails between the values of $P_{p}$, listed under columns 2 and 3 , for the same magnification.

\section{Platinum Wire as Target and 71l-mm Objective in Viewing Telescope}

The target is a platinum wire mounted vertically in the focal plane of the collimator. The 
diameter of the wire is 0.0005 in., which subtends an angle of 4 seconds at the objective. All other conditions of the experiment are essentially the same as those for the spider fiber as target.

The results of measurement are listed in table 2. Assuming the same form of equation connecting $P E_{s}$ and magnification evaluation of the constants $A$ and $B$ by least-squares gives the relation

$$
P E_{s}=\frac{5.262}{M}+0.060
$$

which is nearly the same as eq 4 found for the spider-fiber target. A comparison of observed and calculated values of $P E_{s}$ given in table 2

TABLE 2.-Probable error of a single pointing $\left(P E_{s}\right)$ as a function of magnification $(M)$.

The results are for viewing telescope with an objective of $711-\mathrm{mm}$ focal length, 100 -mm aperture. A platinum wire 0.0005-in. in diameter mounted in the focal plane of the collimator is the target.

\begin{tabular}{|c|c|c|c|c|c|c|c|}
\hline \multirow{2}{*}{ Magnification } & \multicolumn{2}{|c|}{$P E_{s}$} & \multirow{2}{*}{ 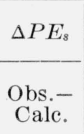 } & \multicolumn{3}{|c|}{$P_{p}$} & \multirow{2}{*}{$\begin{array}{l}\text { Num- } \\
\text { ber of } \\
10^{-} \\
\text {groups }\end{array}$} \\
\hline & Obs. & Calc. & & 1 & 2 & 3 & \\
\hline Diameters & $s e c$ & $s e c$ & $s e c$ & $s e c$ & sec & $s e c$ & \\
\hline $6.0 \ldots \ldots$ & 0.942 & 0.937 & 0.005 & 0.033 & 0.067 & 0.082 & 20 \\
\hline $19.6 \ldots$ & .308 & .328 & -.020 & .011 & .022 & .030 & 20 \\
\hline $25.5 \ldots$ & .270 & .266 & .004 & .007 & .020 & .016 & 40 \\
\hline $36.4 \ldots \ldots$ & .192 & .205 & -.013 & .007 & .014 & .014 & 20 \\
\hline $50 \ldots \ldots$ & .170 & .165 & .005 & .008 & .012 & .011 & 10 \\
\hline $83 \ldots$ & .135 & .123 & .012 & .007 & .010 & .010 & 10 \\
\hline $115 \ldots$ & .105 & .106 & -.001 & .005 & .008 & .009 & 10 \\
\hline $181 \ldots$ & .087 & .089 & -.002 & .004 & .006 & .006 & 10 \\
\hline $223 \ldots$ & .094 & .084 & .010 & .005 & .007 & .012 & 10 \\
\hline $253 \ldots$ & .079 & .081 & -.002 & .004 & .006 & .008 & 10 \\
\hline $414 \ldots$ & .070 & .073 & -.003 & .004 & .005 & .005 & 10 \\
\hline
\end{tabular}

shows excellent agreement. The significance of $\triangle P E_{s}$ and the three values of $P_{p}$ are the same as explained in section 1 . The theoretical value of $P_{p}$, listed in column 2 , shows very close agreement with the value of $P_{p}$ determined from the spread of values of $P E_{s}$, listed under column 3.

\section{Platinum wire as target and 1,143-mm objective in viewing telescope}

In this part of the experiment, a telescope objective with focal length of 1,143-mm is used to study possible effect on the values of $P E_{s}$ that may arise with change of focal length of the observing telescope objective. All other conditions remain the same as described in section 2 .

The results of measurements are listed in table
3. Assuming that the form of the equation connecting $P E_{s}$ and magnification is the same as eq 3 , evaluation of the constants by least-squares gives the relation

$$
P E_{s}=\frac{4.592}{M}+0.067
$$

which closely resembles eq 4 and eq 7. A comparison of observed and calculated values given in table 3 shows excellent agreement.

TABLE 3.-Probable error of a single pointing $\left(P E_{8}\right)$ as a function of magnification $(M)$.

The results are for a viewing telescope with an objective of $1,143-\mathrm{mm}$ focal length, aperture 50-mm. A platinum wire of 0.0005-in. in diameter mounted

\begin{tabular}{|c|c|c|c|c|c|c|c|}
\hline \multirow{2}{*}{ Magnification } & \multicolumn{2}{|c|}{$P E_{8}$} & \multirow{2}{*}{ 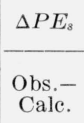 } & \multicolumn{3}{|c|}{$P_{p}$} & \multirow{2}{*}{$\begin{array}{c}\text { Num- } \\
\text { ber of } \\
10- \\
\text { groups }\end{array}$} \\
\hline & Obs. & Calc. & & 1 & 2 & 3 & \\
\hline Diameters & $\sec$ & $\sec$ & $\sec$ & $\sec$ & $\sec$ & $\sec$ & \\
\hline $26.5 \ldots$ & 0.239 & 0.240 & -0.001 & 0.017 & 0.017 & 0.017 & 5 \\
\hline $32.2 \ldots$ & .208 & .210 & -.002 & .015 & .015 & .024 & 5 \\
\hline $49.1 \ldots$ & .165 & .161 & .004 & .012 & .012 & .017 & 5 \\
\hline $134 \ldots$ & .100 & .101 & -.001 & .007 & .007 & .008 & 5 \\
\hline $157 \ldots$ & .099 & .096 & .003 & .007 & .007 & .007 & 5 \\
\hline 291 & .079 & .083 & -.004 & .006 & .006 & .012 & 5 \\
\hline
\end{tabular}
in the focal plane of the collimator is the target.

\section{Discussion}

Although the variation of probable error of telescope pointing with magnification has frequently been the subject of investigation [1], the results have been so diverse that it seemed worthwhile to study this phase of pointing accuracy with the present equipment. It seems probable that the wide variations in the results previously reported arise from the attempt to correlate $P E_{s}$ with magnification for pointings made at an outdoor target without control of other variables. Such pointings are likely to be influenced by turbulence of the air intervening between observer and target, lighting conditions and degree of contrast, configuration or shape of the target viewed, arrangement and size of the cross-hairs in the observing telescope, and small movements of the observing telescope or the object viewed. In addition, some investigators have handicapped themselves at the outset by assuming that there should be a definite relation connecting pointing accuracy and resolving power of the telescope objective [6, 7, 8, and 9]. 
The possibility of such a relation is minimized by Tilton [10] who, in the course of some precise work on prism size, found it necessary to study the effect on pointing accuracy with change of aperture of objective. His findings indicate no significant change or pointing accuracy with resolving power. It is probable that a belief (not always definitely stated) that pointing accuracy does not differ greatly from the limit of resolving power influenced some investigators to be satisfied with a relatively coarse adjustment in making their settings. For example, Pelzer [4], the first worker to make definite attempts to control brightness, arrives at such high values of $P E_{s}$ (varying from 0.9 to 1.4 seconds for magnifications varying from 28 to 14 ) that it appears very likely that he did not employ a sufficiently fine means of reading his pointings. Also, many observers appear to base their formulas relating magnification and pointing accuracy upon results obtained for relatively few magnifications. Thus, Noetzli [11], in his work on outdoor pointing, bases his equation

$$
P E_{s}=\frac{2.7}{\sqrt{M}} \text { to } \frac{2.0}{\sqrt{M}}
$$

upon magnifications 12, 24, and 37, while Klempau [12] bases his equation

$$
P E_{s}=\frac{4.9}{\sqrt{M}} \text { to } \frac{3.0}{\sqrt{M}}
$$

upon magnifications 19,23 , and 30 .

One of the best pieces of work definitely dealing with indoor pointing accuracy is that by Noetzli [11]. He first investigates the effect of magnification upon pointing accuracy under conditions that preclude errors arising from the presence of cross-hairs in the ocular, and, for a range of magnification from 1 to 78 , finds the relation ${ }^{2}$

$$
P E_{s}=\frac{1.39}{M}
$$

However, in making the settings in the second part of his investigation, wherein he used crosshairs in the ocular, he gets the relation

$$
P E_{s}=\frac{0.486}{\sqrt{M}},
$$

${ }^{2}$ The constants in Noetzli's equations have been adjusted by the present author to express probable error instead of root-mean-square error. which is clearly open to question in view of the present study. In seeking an explanation for this discrepancy, the present author has plotted Noetzli's values in the form of $M \times P E_{s}$ versus $M$, which is shown in figure 4, for pointings both with and without cross-hairs. It is clear for those values of $M$ in excess of 15.7 that Noetzli's values with cross-hairs fall on a curve satisfying the equation

$$
M \times P E_{s}=1.665+0.035 M,
$$

while those values of $M \times P E_{s}$ found for $M$ varying from 1 to 8.6 not only fall well below this curve, but they also are lower than the corresponding values of $M \times P E_{s}$ for all magnifications without cross-hairs. It seems very improbable that the pointings with cross-hairs should be better than the pointings without cross-hairs for magnifications 1 to 8.6 and inferior for magnifications in excess of 15.7. It is noteworthy that these discrepancies are pronounced only for the lower magnification where, according to table 1 in Noetzli's paper, a telescope objective was used as ocular. Whenever long focal length lenses are used as oculars, it is possible that there may be sufficient difference in adjustment of objective and ocular (or of the reticle with respect to the objective and ocular) to produce a difference in magnification between the condition of pointing with and without cross-hairs. The tendency for the normal observer would be toward an adjustment producing a higher magnification for pointings with than for those without cross-hairs. Accordingly, the magnifications 1 to 8.6 , listed by Noetzli, may actually have been somewhat higher for the condition of pointing with crosshairs that they were without cross-hairs.

When eq 13 is transformed into the equivalent form

$$
P E_{s}=\frac{1.65}{M}+0.035
$$

which is identical with the form in eq 1 , it is clear that the constant $B$ is not appreciably different from the average value of 0.068 found in the present experiment. The value $A$ is, however, very much smaller than the value of 4.962 reported here. Since, however, the constant $A$ is the vernier acuity of the observer's eye, and there is considerable variation in this quantity from one 


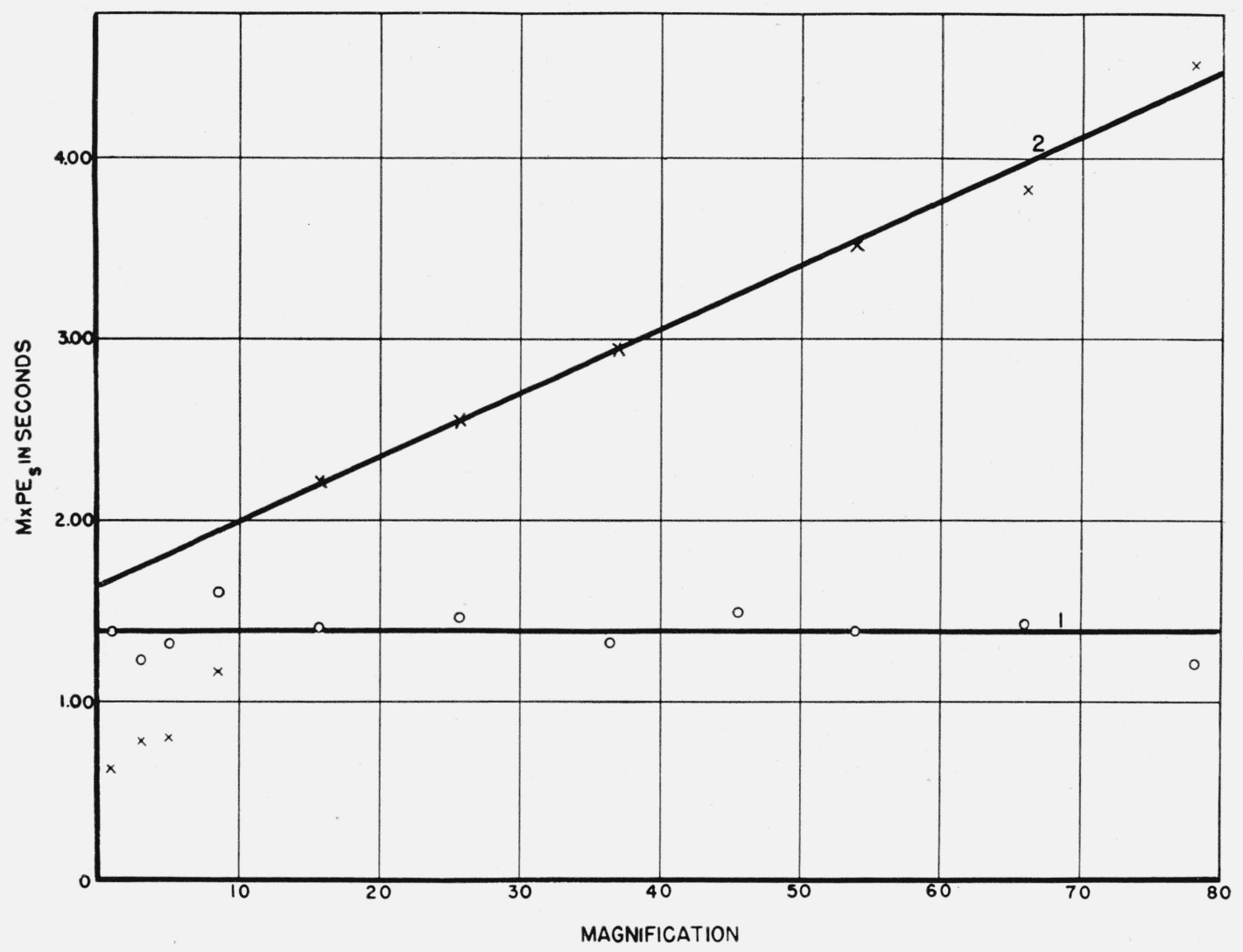

Fig URE 4.- $M \times P E_{\mathrm{s}}$ versus $M$.

Observed values of $M \times P E$, with cross-hairs are indicated by crosses; curve 2 shows the theoretical curve for $M>15.7$. Observed values of $M \times P E$, with. out cross-hairs are indicated by circles; curve 1 shows the theoretical curve. These results are obtained from Noetzli's paper. (See reference [11]).

observer to another, the differences in the constant $A$ introduce no difficulty.

\section{Conclusion}

The relation between $P E_{s}$ and magnification is found from this experiment to be expressible in the form

$$
P E_{s}=\frac{A}{M}+B
$$

where $A$ and $B$ are constants. On averaging the values of $A$ and $B$ for the three cases described, the resulting equation is

$$
P E_{s}=\frac{4.962}{M}+0.068
$$

It is problematical whether any significance can be attached to the variation of the constants $A$ and $B$ for the three cases. This question can only be answered by further study where a wider range of target diameters and focal lengths of the observing telescope are used. The constant $A$ is the vernier acuity of the observer's eye and can undoubtedly be expected to vary from one observer to another. The value of 4.96 seconds is within the range of values obtained by different observers, contained in a discussion of vernier acuity in a paper by Walls [13].

It is more difficult to account for the constant $B$. Previous workers in this field have used equations in which $A$ only appeared, $P E_{s}$ being correlated with the reciprocal of magnification. Two possibilities are here advanced in interpretation of the constant $B$. The first is vibration that is always present however much it may be reduced by careful mounting. The second possibility is that the short air column separating the collimator and telescope objective may by its turbulence introduce the additional error represented in the equation by $B$. In the study of 
outdoor pointing, the possibility of a dependence of pointing accuracy upon range was discussed [1]. The contribution dependent on range was given as $0.064 d^{\frac{1}{4}}$ where $d$ is the range in meters. The distance separating the collimator and telescope objective in this experiment was approximately 0.75 meter. Accordingly the computed error arising from range is approximately 0.059 second, which is not significantly different from the values of $B$ found in the present experiment on indoor pointing.

\section{References}

[1] F. E. Washer and H. B. Williams, Precision of telescope pointing for outdoor targets. J. Optical Soc. Am. 36, 400 (1946).

[2] F. E. Washer and H. B. Williams, precision of telescope pointing for outdoor targets. J. Research NBS 36, 479 (1946) RP1717.

[3] J. Guild, The insensitivity and personal equation errors of optical settings. Trans. Optical Soc., (London) 31, 1 (1929-30).

[4] A. Pelzer, Uber den Einfluss der Lichtstarke von Theodolit- und Nivellier-fernrohren auf den mittleren Zielfehler, Z. Instrumentenk. 46, 354 (1926).
[5] A. G. Worthing and J. Geffner, Treatment of experimental data, p. 197 (John Wiley \& Sons, Inc., New York, N. Y., 1943).

[6] A. A. Michelson, Measurement by light-waves. Am. J. Sci. 39, 115 (1890).

[7] A. A. Michelson, Light-waves and their application to metrology. Nature 49, 56 (1893-94).

[8] F. L. O. Wadsworth, On the conditions which determine the ultimate optical efficiency of methods for observing small rotations. Phil. Mag. 44, 83 (1897).

[9] F. L. O. Wadsworth, On the optical conditions required to secure maximum accuracy of measurement in the use of telescopes and spectroscope. Astrophys. J. 16, 267 (1902).

[10] L. W. Tilton, Prism size and orientation in minimum deviation refractometry. BS J. Research 6, 59 (1931) RP262.

[11] A. Noetzli, Untersuchung uber die Genauigkeit des Zielens mit Fernrohren. Z. Instrumentenk. 35, 65 and 89, (1915).

[12] Fr. Klempau, Uber die Beziehungen zwischen WinkelNonien-, und Zielgenauigkeit, Allgem. Verm.Nachr. 25, 317 (1923).

[13] G. L. Walls, Factors in human visual resolution, J. Optical Soc. Am. 33, 487 (1943).

Washington, October 2, 1946 